\title{
Comparison of typical hydro governor models for the study of power system frequency characteristics
}

\author{
Ya-Wei Du ${ }^{1}$, Qing Wang, ${ }^{2, *}$, Chang-Ming Jiang ${ }^{3}$, \\ Ya-Zhou LUO ${ }^{3}$, Yue-Qiao Li ${ }^{1}$, Peng ZI ${ }^{3}$, Yun-Ting Song ${ }^{2}$ \\ 1.North China Electric Power University, \\ Changping District, China \\ †E-mail:627975708@qq.com \\ 2.China Electric Power Research Institute, \\ Haidian District, Beijing, China \\ E-mail: wangqing@epri.sgcc.com.cn \\ 3.North China Branch of State Grid Power Dispatch \& Control Center, \\ 100053, Beijing, China
}

\begin{abstract}
In this paper, the model of hydro governor used in power system stability calculation is analyzed. According to the traditional model and the characteristics of the dead zone of the modern digital electro - hydraulic model, the effect of the dead zone on the frequency modulation effect is studied. The important parameters (Frequency coefficient) of the modern electric hydraulic model of hydraulic turbine are given in detail; The common software (PSD-BPA), which is used in power system stability calculation, is analyzed from the angle of the maximum frequency deviation, the stability time and the output power of the generator, and according to different disturbance conditions, analysis of the various types of speed control model of the applicable conditions. The simulation results show that the power imbalance caused by the system fault is in the primary frequency modulation limit, the modern digital electro hydraulic speed control model has better speed control effect, and vice versa. In this paper, the application of speed governing model and some parameters setting of the speed control model are provided for the practical engineering application of hydraulic turbine governing model.
\end{abstract}

Keywords: hydraulic turbine; speed governing model; the limiting of Primary frequency modulation.

\section{Introduction}

Chinese from 1950s began to design and production of the governor, after three generations, the governor also from the original mechanical hydraulic type (MHC), after the electric hydraulic (EHC) improvement, finally in the domestic control work load modeling developed digital electrical hydraulic control system (DEH) [3-5], governor technology in reliability and the adjustment function, 
regulation and other aspects of quality are greatly improved.

At present, the domestic production of new digital electro-hydraulic governor, has been quite common[5-6], but in the actual engineering of transient stability calculation, the traditional control model of hydraulic turbine recommended by IEEE in 1973[8] is still used in most parts of the country, namely mechanical hydraulic speed control model, the reason is that although in recent years the domestic of generator, excitation system and load modeling and the parameter measuring work is positive progress, but calculated by the governor on the stability of the power system modeling and parameter identification of less simulation model and the actual operation of the governor in the structure and the parameter setting is still a big difference [9-13].

In view of this, this paper uses the PSD-BPA control model of hydropower, different characteristics of in-depth analysis of the models, The setting method of key parameters such as adjustment coefficient is put forward, and the application of all kinds of speed control model is studied under different disturbance conditions.

\section{Major Headings Comparison of Speed Control Model of Hydraulic Turbine}

The traditional hydraulic turbine governor model also known as the mechanical model, the digital electro-hydraulic turbine speed control model (hereinafter referred to as the DEH model) is widely used in the engineering project.

\subsection{Comparison of model structure}

In 1973, the IEEE Committee recommended the turbine's traditional governing model, which includes two parts: the pressure valve and the guide vane relay. The structure is shown in Figure 1. According to the rotor motion equation of generator set, the formula can be listed (1). When the system is disturbed, the generator mechanical power is not equal to the electromagnetic power will produce the frequency deviation [13], frequency deviation after pressure valve and the guide vane servomotor is eventually converted into PGV (valve opening signal), as shown in (2).

$$
J \frac{d \omega}{d t}=P_{M}-P_{E}
$$

Note: $\omega$ for the generator speed; $J$ for the generator inertia time constant; $P_{\mathrm{M}}$ for the mechanical power; $P_{\mathrm{E}}$ for the electromagnetic power. 


$$
\mathrm{P}_{\mathrm{GV}}=\frac{\mathrm{P}_{0} \mathrm{R}-K^{\prime} \Delta \omega}{\varepsilon_{1}+\varepsilon_{2}+S^{2} T_{G} T_{P}+S T_{G}}
$$

Note: $T_{\mathrm{G}}$ for governor response time; $T_{\mathrm{P}}$ for the pilot valve time constant; $\mathrm{s}$ for the Laplasse operator; $\varepsilon_{1}=\frac{S D_{d} T_{d}}{1+S T_{d}}$,for soft feedback coefficient; $\varepsilon_{1}=R$, hard feedback coefficient; $\mathrm{K}$ ' for Amplification factor of rotational frequency deviation signal

Figure 2 is the typical structure of DEH speed governing system of hydraulic turbine which is already very popular at present [14]. The model contains proportional plus integral plus derivative(PID). The amount of feedback in the closed-loop control of electromagnetic power PE and gateopening (YPID) constitute a switch between two modes of adjustment.

The input variable of the traditional speed control model only has the frequency deviation, and the DEH speed regulation system has many kinds of modes. The input signal can be the frequency deviation + the generator mechanical power, the frequency deviation + the open degree signal or the DEH open loop mode, The regulation law of generating set follows the following formula.

$$
P_{M}=f\left(\Delta \omega, \Delta P_{E}, \Delta Y\right)
$$

Note: $\omega$ in the formula for the speed change, PE for the generator electromagnetic power change, $\quad \mathrm{Y}$ for the degree change.

Both the traditional speed regulation model and the DEH model have feedback link. The traditional control model is the relay output valve opening signal as the feedback, divided into soft and hard part two feedback and both feedback as the input signal of the pilot valve, The feedback span is large, and the input of the speed control model has a certain time delay. However, DEH speed control system only has the feedback link in the open degree model. In this mode, the output of the PID controller PGV feedback loop back to the integral part of the PID controller, the feedback span is small, almost no delay.

Because of the early mechanical governor structure, the traditional speed control model is relatively simple, only the pilot valve and the relay link has a limited range. The DEH speed control system and electro-hydraulic servo system has a frequency modulation, opening, integration, power, sub cycle PID control link output, regulator output, guide vane relay speed and valve position eight kinds of limit. Among them, the limit of the speed of the guide vane relay and the limit of the output of the PID control link can be obtained according to 
the field test, which is more in line with the actual requirements in the transient stability calculation.

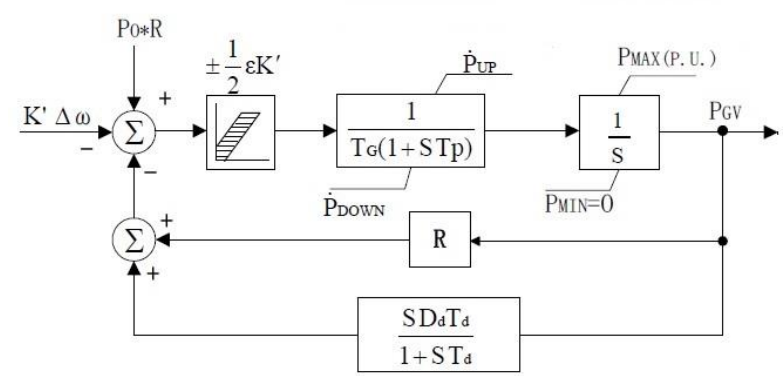

Fig. 1 Model of traditional hydraulic turbine governor

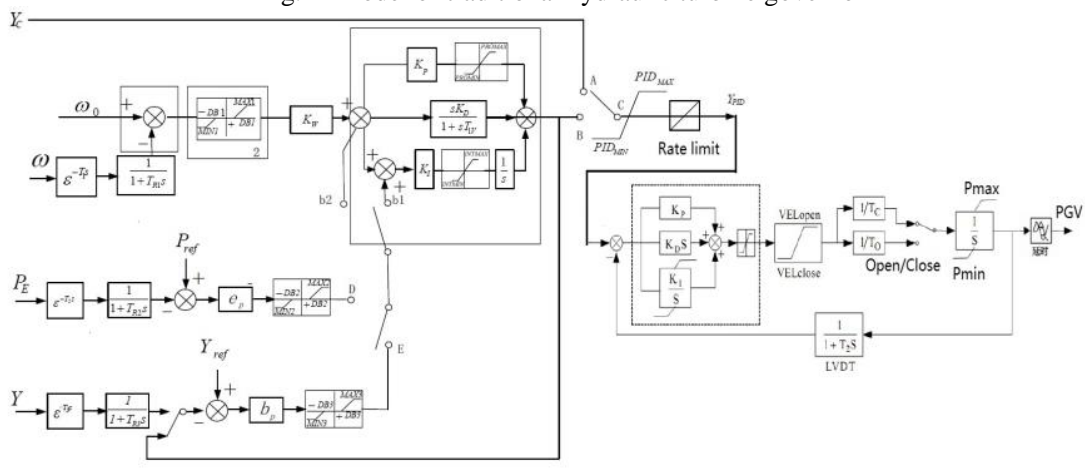

Fig. 2 Model of traditional hydraulic turbine governor

\subsubsection{Sub-subheadings}

Typeset sub-subheadings in medium face italic and capitalize the first letter of the first word only. Section numbers to be in roman.

\section{Hydraulic Turbine Governor Dead Zone}

\subsection{Importance of Dead zone Comparison of dead zone}

In the calculation and Simulation of dead zone is often necessary but easy omissions, dead time settings will cause the inaccurate calculation results and actual errors.

Because the traditional model belongs to the old mechanical model, there is only the inherent dead zone. In the DEH model, the frequency of the dead zone is the integrated [14] of the inherent speed dead zone and the artificial frequency dead zone.

So how to distinguish the difference between the two models of the dead 
zone and set the dead zone correctly to achieve the ideal simulation results will be analyzed in this paper.

\subsection{Comparison of dead zone}

As shown in Figure 1, the conventional hydraulic turbine speed control model only contains the inherent dead zone, This kind of dead zone is the sum of the deviation among valve lap, reverse drag of driving mechanism and others. So in the traditional control model in the dead zone input should include the frequency deviation and gateopening deviation (with feedback input). And achieve steady state when the valve position is no longer change, that is, the input signal of the oil motivation is zero. When the input signal of the dead zone is in the dead zone, it can reach the steady state, so it can be set up according to the stable condition:

$$
-\frac{1}{2} \varepsilon K^{\prime} \leq-K^{\prime} \Delta \omega+\left(P_{0}-P G V\right)^{*} R \leq \frac{1}{2} \varepsilon K^{\prime}
$$

Note: $P_{0}$,target power; $\mathrm{PGV}$, the valve opening degree;

$$
R=-\frac{\Delta f / 50}{\Delta P}
$$

Equation (5) is used to calculate the adjustment coefficient.

0p.u.,0.001p.u., 0.002p.u., respectively, the dead zone of the conventional speed control model of hydraulic turbine, the other parameters are unchanged, condition such as the third chapter describes the small disturbance. The simulation results are shown in Figure 3. The input signal of the dead zone is the sum of the three(the frequency deviation,valve position feedback and target power), so the frequency deviation is only a small part, so the change of the dead zone almost does not affect the steady state frequency deviation, only affects the adjustment effect in the dynamic process. As shown in Figure 2, the DEH model of the hydraulic turbine is mainly generated in the frequency measurement link, that is, the artificial dead zone. The dead zone setting affects only the frequency deviation signal, the frequency deviation of the amplification coefficient of $1 / R$ is relatively large, so the dead changes not only influence the dynamic adjustment effect, influence on frequency is also very obvious. 


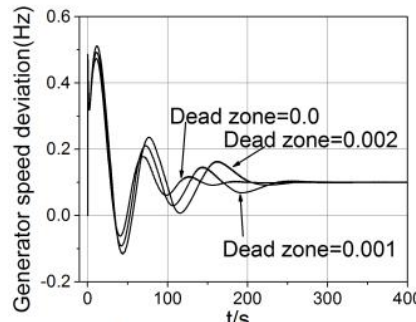

(a) Traditional governor model

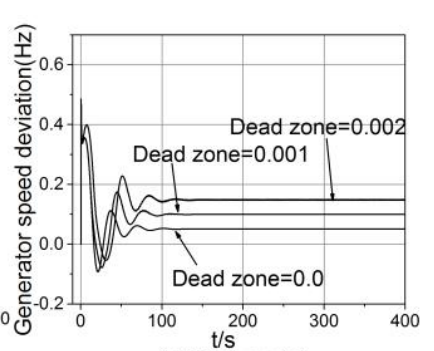

(b) DEH mode

Fig. 3 Dead zone contrast curve of speed regulation model

\subsection{Setting of Adjustment Coefficient}

The adjustment coefficient of the traditional turbine governing model can be set directly, and the adjustment coefficient of the DEH model is not directly given, so it is to be calculated by the steady condition.

According to the traditional model, when the adjustment coefficient $\mathrm{R}=0.05$, DEH model for the power mode, set the adjustment coefficient.

Under small disturbance conditions, the load node has a three phase short circuit fault in 0.1 seconds, and the fault line is removed in 0.2 seconds, the turbine adopts the traditional speed regulation model. The simulation results show that the steady state generator frequency deviation is $0.01 \mathrm{HZ}(0.002 \mathrm{pu})$, the generator mechanical power deviation is $-12 \mathrm{MW}(-0.04 \mathrm{pu})$, according to the equation (5) can get $\mathrm{R}=0.05$, consistent with set values. This can be used as the basis for setting the adjustment coefficient of the DEH model.

According to the DEH model, the condition of steady state can be reached:

$$
\left(-\Delta \omega^{+} \varepsilon\right) * K_{W}+\Delta P_{E} * e_{P}=0
$$

Take $K_{\mathrm{w}}=1$, get $e_{\mathrm{p}}=0.025$.

This is the corresponding parameter when the adjustment coefficient $\mathrm{R}=0.05$ is in this condition.

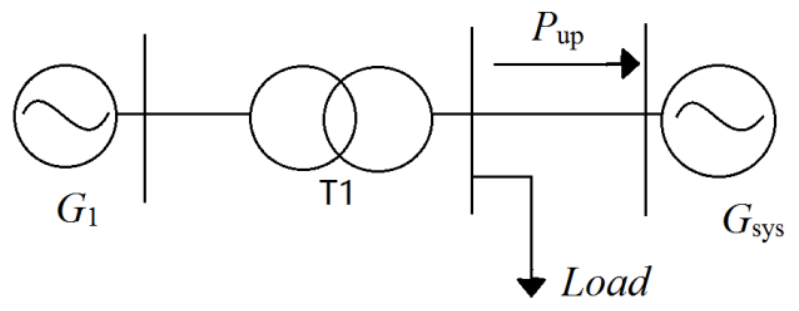

Fig. 4 The diagram of large unit connected to infinite bus system 


\section{Simulation Results and Analysis}

A single machine infinite bus model is established in PSD-BPA, simulate the scenario of generating excess power with the fault of the contact line. The rated capacity of the unit is $300 \mathrm{MW}$, and the system structure is shown in Figure 4.

As the standard provisions of the general primary frequency regulation is set to the rated load of the unit $\pm 6 \% \sim \pm 10 \%$. So in this paper, we use two kinds of working conditions, which are big disturbance and small disturbance.

Under small disturbance conditions, the load nodes absorb active power 16.3MW, which accounts for $5.4 \%$ of the total power generation.

Under large disturbance conditions, the load node absorbs active power 33.3MW, which accounts for $11.1 \%$ of the total power generation.

\subsection{Simulation Results}

Both models' adjustment coefficient $\mathrm{R}=0.05$, dead zone 0.001 . Other parameters of DEH hydraulic turbine governing model adopt field measured parameters. Typical parameters are adopted for other parameters of the traditional hydraulic turbine governing model. The simulation results are shown in Figure 5, the peak of the rotational speed deviation, the stable time and the final steady state frequency deviation are shown in table 1.

TABLE I. COMPARISON RESULT OF SPEED REGULATION EFFECT

\begin{tabular}{|c|c|c|c|c|}
\hline Conditions & Model & $\begin{array}{c}\text { Maximum } \\
(\mathrm{Hz})\end{array}$ & $\begin{array}{c}\text { Steady time } \\
(\mathrm{s})\end{array}$ & $\begin{array}{c}\text { Steady } \\
\mathrm{f}(\mathrm{Hz})\end{array}$ \\
\hline \multirow{3}{*}{ Small } & traditional & 0.552 & 265 & 0.0996 \\
\cline { 2 - 5 } & DEH & 0.356 & 140 & 0.1 \\
\hline \multirow{3}{*}{ Large } & traditional & 1.175 & 310 & 0.235 \\
\cline { 2 - 5 } & $\mathrm{DEH}$ & 1.545 & 150 & 0.235 \\
\hline
\end{tabular}

\subsection{Analysis of Simulation Results}

After the disturbance, the water hammer effect is occurred in the turbine prime mover of the conventional speed control model and the DEH model. As shown in Figure 5 (a), 5 (c), in the load rejection moment, the turbine prime mover output PM will be instantaneous increase, the mechanical power of the generator and the electromagnetic power imbalance. As shown in Figure 5 (f), the PGV curve does not rise at the beginning of the disturbance, indicating that the effect is only the original motivation. 


\section{1)Small Disturbance}

The DEH model is more effective in suppressing the frequency to increase after the small disturbance. As shown in Figure 5(a), (b), due to the DEH model only in the frequency measurement link, oil motivation to open / close links have a time delay, So the traditional model can more quickly control the guide vane servomotor with respect to, and with the help of PID control, DEH can control a greater degree of gateopening.

According to the table I can be seen, the traditional model of small disturbance to achieve stable stage longer. In the traditional model, the delay of the pilot valve is larger, the proportion of soft feedback is larger, and there is a certain delay, resulting in over modulation phenomenon. Eventually lead to the mechanical power of the generator after many times to reach stability.

\section{2)Large Disturbance}

In the simulation of large disturbance, DEH control ability of a vane is suppressed in the adjustment phase. As shown in Figure 5(e), the DEH model of the PID input signal in the $0.2 \mathrm{~s}-68.77 \mathrm{~s}$ and $83.9 \mathrm{~s}-120.5 \mathrm{~s}$ to achieve the limit of the lower limit, indicating that the speed control model to achieve a frequency modulation limit. At this point, the input signal of the frequency deviation of the generator reaches the limit, the output of the PID controller is changed to a fixed value, and the output of the integral link is changed only by the variation of the electromagnetic power deviation. So as shown in Figure 5 (d), the output of the PM between the 0.2 68.77s is a smooth curve. 




(a) Generator speed deviation under small disturbance

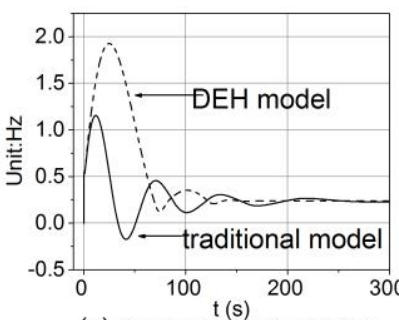

(c) Generator speed deviation under large disturbance

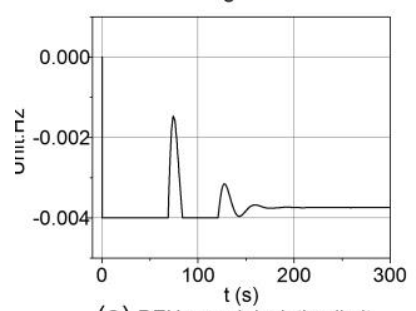

(e) DEH speed deviation limit under large disturbance

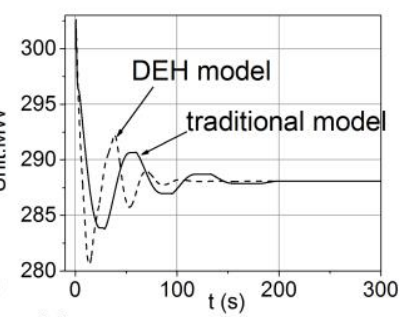

(b) Mechanical power of generator under small disturbance

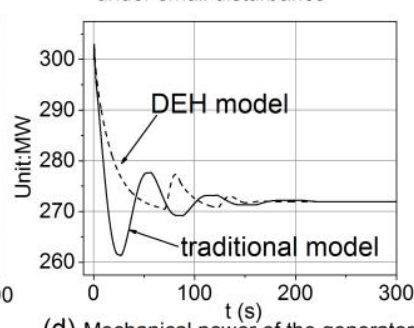

(d) Mechanical power of the generato under large disturbance

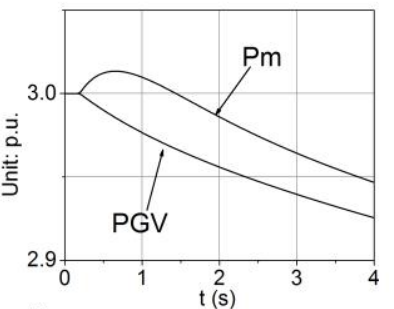

(f) Gateopening signal and output signal

FIG. 5 SIMULATION RESULTS OF HYDRAULIC TURBINE SPEED CONTROL MODEL

Due to the role of the proportion of restrictions, so that the speed of the reaction is not sensitive, resulting in a decline in the output curve of the generator is behind the traditional model curve.

At the same time the input signal strength of the whole controller weakened, opening limitation of turbine guide, eventually led to the generator output curve under small potential.

After the output is restored to the limit, the DEH model is stable and the DEH model is stable as long as the feedback delay is small.

\section{Conclusion}

1)At present, the governing model of hydraulic turbine used in engineering project is divided into the traditional model and the digital electro hydraulic type. In the structure of the DEH model is relatively complex, and has a variety of 
models, each part of the set more in line with the actual speed control system.

2)The dead zone setting of the traditional speed control model of hydraulic turbine only affects the effect of dynamic adjustment. The DEH model of turbine is not only affected by the dead zone, but also has a certain effect on the steady state frequency deviation.

3)This paper presents a method to accurately set the adjustment coefficient of the DEH model of the turbine. In depth analysis of the application of speed control model under different conditions. The simulation results show that the load rejection rate is lower than that of primary frequency modulation, and the DEH model is better than the traditional model, and the load rejection rate is more than one time, and the frequency modulation effect is more ideal.

\section{Acknowledgements}

This work is supported by State Grid Corporation of China(No. XT71-16043).

\section{References}

1. Shao Yixiang, Cai Xiaofeng, Cai Weijiang, "A review of the technical development of the hydraulic turbine governor in China," Hydropower automation and dam monitoring, 2009, 33 (4): 16-19.

2. Kim Na, Liu Wenying, Cao Cao, "Effects of primary frequency modulation parameters of large capacity units on the frequency characteristics of power grid," Power system protection and control, 2012, 40 (1): 91-95.

3. I. S. Jacobs and C. P. Bean, "Fine particles, thin films and exchange anisotropy," in Magnetism, vol. III, G. T. Rado and H. Suhl, Eds. New York: Academic, 1963, pp. 271-350. Xiao Zenghong, Xu Feng, "Digital electro hydraulic control system for steam turbine, "China Electric Power Press, 2003.

4. Zhang Suxin, Yang Qiguo, Wang Weimin, "The development and Prospect of the steam turbine industry in China," Thermal Turbine, 2003(1):1-5.

5. Zhao Kunyao, Yang Huisheng, "Development situation of hydraulic turbine governor technology in China," Water conservancy and Hydropower Technology, 1996(05):49-51.

6. Zhou Jian, Li Gang, Zhu Ruijin, "Study on the model of hydro turbine governing system applied to the stability calculation of power system," East China electric power, 2008, 36 (07): 76-80.

7. Shanghai Xinhua Control Technology (Group) Co., Ltd, "Digital electro hydraulic control system for steam turbine in power plant -DEH, " China Electric Power Press, 2005. 
8. Report I C. Dynamic Models for Steam and Hydro Turbines in Power System Studies[J]. IEEE Transactions on Power Apparatus \& Systems, 1973, PAS-92(6):1904-1915.

9. Li Hua, the history of Keqin, fan Yue, "Structural analysis of hydraulic turbine governor model for stability calculation of power system" Power system technology, 2007, 31 (05): 25-28.

10. Jiang Lei, Zhu Shouzhen, "Four parameters database of Northeast Power Grid Based on parameter identification technology,"Northeast China electric power technology, 2002, 23 (12): 45-48.

11. Ju Ping, Chen Xinqi, "Simulation evolution method for parameter identification of synchronous generator, " Chinese Journal of electrical engineering, 1999, 14 (05): 1-4.

12. He Renmu, "Research on the accuracy of power system dynamic simulation," Power system technology, 2000, 24 (12): 1-4.

13. He Ren mu, Wang Weiguo, Jiang Debin, "Research on the measurement model of dynamic load and the validity of model in Guangdong power grid, " Chinese Journal of electrical engineering, 2002, 22 (03): 78-82.

14. Xi'an Jiao Tong University, "Power system calculation, "Water conservancy and electric power press, 1978.

15. $\mathrm{Xu}$ Guangwen, "PSD-BPA model of hydraulic turbine governor and its application," Guangdong electric power, 2014, 27 (05): 34-38. 